\title{
Pulse carving using nanocavity-enhanced nonlinear effects in photonic crystal Fano structures
}

Bekele, Dagmawi Alemayehu; Yu, Yi; Hu, Hao; Guan, Pengyu; Ottaviano, Luisa; Galili, Michael; Oxenløwe, Leif Katsuo; Yvind, Kresten; Mørk, Jesper

Published in:
Optics Letters

Link to article, DOI:

10.1364/OL.43.000955

Publication date:

2018

Document Version

Peer reviewed version

Link back to DTU Orbit

Citation $(A P A)$ :

Bekele, D. A., Yu, Y., Hu, H., Guan, P., Ottaviano, L., Galili, M., Oxenløwe, L. K., Yvind, K., \& Mørk, J. (2018). Pulse carving using nanocavity-enhanced nonlinear effects in photonic crystal Fano structures. Optics Letters, 43(4), 955-958. https://doi.org/10.1364/OL.43.000955

\section{General rights}

Copyright and moral rights for the publications made accessible in the public portal are retained by the authors and/or other copyright owners and it is a condition of accessing publications that users recognise and abide by the legal requirements associated with these rights.

- Users may download and print one copy of any publication from the public portal for the purpose of private study or research.

- You may not further distribute the material or use it for any profit-making activity or commercial gain

- You may freely distribute the URL identifying the publication in the public portal 


\title{
Pulse carving using nanocavity-enhanced nonlinear effects in photonic crystal Fano structures
}

\author{
Dagmawi A. Bekele, Yi Yu, Hao Hu, Pengyu Guan, luisa Ottaviano, Michael Galili, Leif \\ Katsuo OxenløWe, Kresten Yvind, and Jesper Mork*
}

DTU Fotonik-Department of Photonics Engineering, Technical University of Denmark, DK-2800, Kgs. Lyngby, Denmark

*Corresponding author: jesm@fotonik.dtu.dk

Compiled January 16, 2018

We experimentally demonstrate the use of a photonic crystal Fano resonance for carving-out short pulses from long-duration input pulses. This is achieved by exploiting an asymmetric Fano resonance combined with carrier induced nonlinear effects in a photonic crystal membrane structure. The use of a nanocavity concentrates the input field to a very small volume leading to an efficient nonlinear resonance shift that carves a short pulse out of the input pulse. Here, we demonstrate shortening of $\sim 500$ ps and $\sim 100$ ps long pulses to $\sim 30 \mathrm{ps}$ and $\sim 20 \mathrm{ps}$ pulses respectively. Furthermore, we demonstrate error-free low duty cycle return-to-zero (RZ) signal generation at $2 \mathrm{Gbit} / \mathrm{s}$ with energy consumption down to $\sim 1 \mathrm{pJ} / \mathrm{bit}$ and power penalty of $\sim 2 \mathrm{~dB}$. The device physics and limitations are analyzed using nonlinear coupled-mode theory. (c) 2018 Optical Society of America

OCIS codes: (130.3120) Integrated optics devices; (320.5540) Pulse shaping; (190.0190) Nonlinear optics; (050.5298) Photonic crystals.

\section{http://dx.doi.org/10.1364/ao.XX.XXXXXX}

Fano resonances appear due to the interaction between a localized mode and a continuum of modes, giving rise to characteristic spectral features [1-3]. Recently, such resonances have found several applications in nanophotonics such as in switching [4], lasing [5], non-reciprocal transmission [6] and sensing [7]. Here, we suggest and demonstrate a new class of applications of Fano resonance for processing pulses, i.e. return-to-zero (RZ) signals encountered in on-chip communication systems. Returnto-zero signals have several advantages over non-return-to-zero (NRZ) signals due to improved receiver sensitivity, less intersymbol interference and lower average power $[8,9]$. For on-chip and chip-to-chip interconnects, larger receiver sensitivity can enable the system to be operated at lower power budget, leading to reduced thermal effects, thus creating opportunities for higher density of integration [8]. Generating short pulses or low duty cycle RZ signals is interesting for applications requiring sharp raising and falling edges such as for synchronization and clocking [8]. Furthermore, a simple pulse-edge detection scheme is desirable for applications that require precise information about the time of arrival of a signal [8]. Conventional pulse carving employs active radio frequency (RF) clock signal for modulation, where the input signal (usually NRZ) is carved into RZ signal $[9,10]$.

In this letter, we show that the use of an asymmetric Fano resonance in combination with strong nonlinear optical effects in a nanocavity allows the carving of a short pulse from a long input pulse without the need for an additional control signal. We demonstrate applications of the device for short-pulse generation and detection of the pulse edge of the input signal.
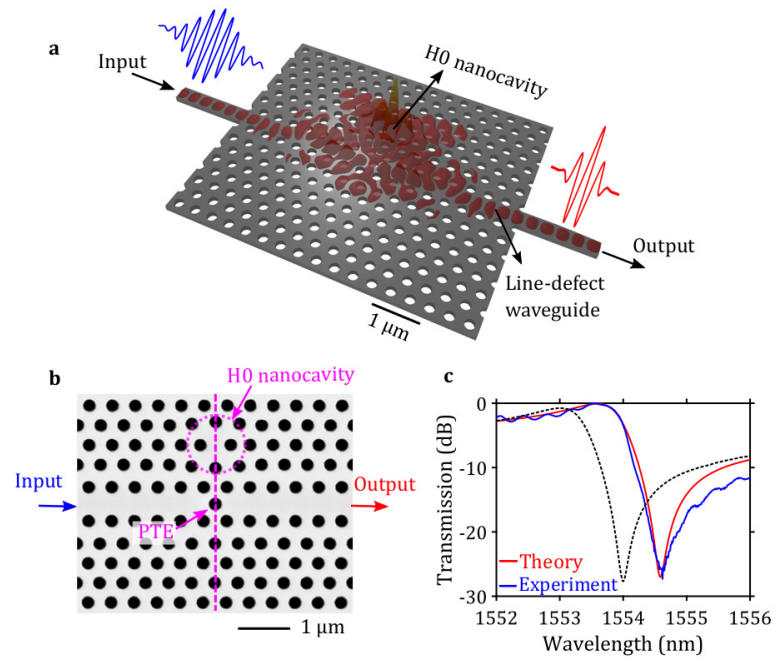

Fig. 1. Photonic crystal (PhC) Fano structure. (a) Device schematic with simulated out-of-plane magnetic field profile. (b) Scanning electron microscope (SEM) image of the fabricated $\mathrm{InP} \mathrm{PhC}$ membrane. It consists of an $\mathrm{H} 0$ nanocavity and a line-defect PhC waveguide. (c) Measured and calculated transmission spectra of the device showing an asymmetric Fano resonance lineshape. The dashed line illustrates the case where the resonance is shifted due to an index change in the nanocavity.

Fig. 1(a) shows a schematic of the device together with a finite-difference time domain (FDTD) simulation of the out-ofplane magnetic field component. The fabricated photonic crystal (PhC) Fano structure, cf. Fig. 1(b), consists of an H0 nanocavity 
[11] side-coupled to a line-defect waveguide. The PhC membrane of thickness $340 \mathrm{~nm}$ is formed by airholes with radius $(r)$ of $125 \mathrm{~nm}$ arranged in a hexagonal pattern with a lattice constant of $447 \mathrm{~nm}$. The $\mathrm{H} 0$ nanocavity is formed by moving a few airholes away from the cavity center while the waveguide is a W1-defect type, albeit with the innermost array of holes being shifted toward the waveguide center [4]. When the discrete mode of the nanocavity and the continuum modes of the $\mathrm{PhC}$ waveguide spectrally overlap, interference occurs, leading to characteristic Fano resonance lineshapes [2, 12]. Importantly, the amplitude of the continuum contribution can be controlled by placing a partially-transmitting element (PTE) in the waveguide [4], which is implemented by having an additional airhole $(r=123 \mathrm{~nm})$ in the waveguide below the nanocavity, Fig. 1(b). This gives rise to a characteristic asymmetric Fano lineshape, see Fig. 1(c). Light is coupled into and out of the device using grating couplers [13].

The dashed line in Fig. 1(c) illustrates the resonance shift due to nonlinear effects in the case of a reduction of the nanocavity refractive index by $\Delta n_{e f f} / n_{e f f} \sim 10^{-3}$, where $n_{\text {eff }}$ is the effective refractive index of the nanocavity. Because of the strongly localized nature of the nanocavity mode and due to its high quality $(Q)$ factor $(\sim 1700)$ and small mode volume, free carriers are efficiently generated in the nanocavity region via two-photon absorption of the input signal $[14,15]$. The change in carrier density in the nanocavity induces a refractive index change and hence resonance shift [16]. Since a large transmission difference between the maximum and minimum points of the Fano resonance is achieved despite a small spectral separation, a slight shift in the resonance spectrum leads to large transmission change.

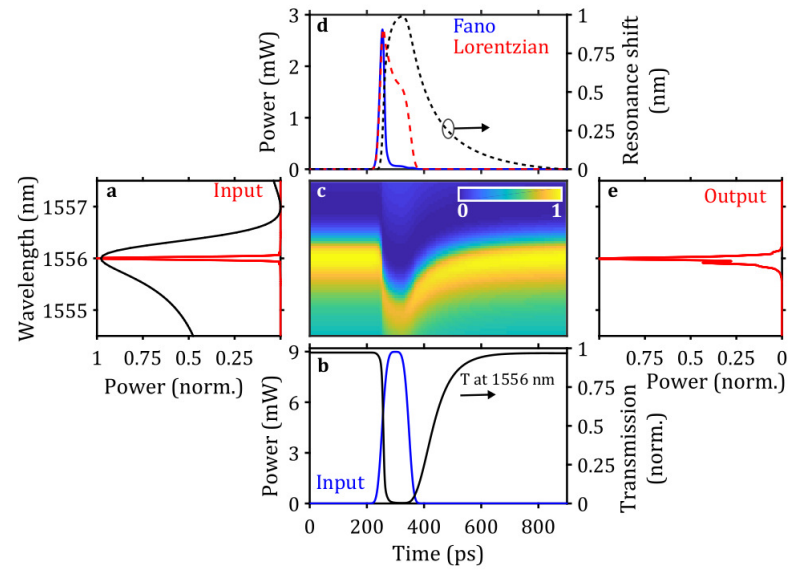

Fig. 2. Basic principle of pulse self-carving using Fano resonance. (a) Fano lineshape (black, linear scale) with resonance peak at $1556 \mathrm{~nm}$ and input pulse spectrum (red). (b) Super Gaussian input pulse (blue) with a width of 93 ps and transmission (black) at $1556 \mathrm{~nm}$ as a function of time. (c) Contour plot of the time evolution of linear transmission spectrum given the input in (a) and (b). (d) Self-carved pulse (blue) with width of $\sim 20$ ps using Fano resonance, partially carved pulse (dashed red) using Lorentzian resonance, and resonance blue shift (dashed black) induced by the input pulse. (e) Spectrum of output pulse.

The basic principle of self-carving using an asymmetric Fano resonance is illustrated in Fig. 2 using coupled-mode theory (CMT) simulations $[17,18]$. For these simulations, most of the material parameters were taken from Ref. [16]. The intrinsic
Q-factor of the nanocavity calculated using FDTD and the total Q-factor estimated from theoretical fits are $1.2 \times 10^{5}$ and 1700 , respectively. In addition, the amplitude transmission coefficient of the PTE is $\sim 0.55$ estimated from fit to the measured resonance transmission. The transmission spectrum of the device shows a Fano resonance (black in Fig. 2(a)), with a transmission peak at $1556 \mathrm{~nm}$. The input signal is a 93 ps long super Gaussian pulse of order 2 and with a peak power of $9 \mathrm{~mW}$. Its temporal pulse shape is shown as the blue curve in Fig. 2(b) and the corresponding spectrum is shown as the red curve in Fig. 2(a), which is centered around the peak of the resonance. The contour plot in Fig. 2(c) depicts the time-evolution of the linear transmission spectrum of the PhC system due to the presence of the input pulse. At 0 ps, the input power of the pulse is zero and the contour plot exhibits the initial transmission spectrum of the system. As the amplitude of the input pulse starts to increase, the output pulse amplitude increases until 255 ps, cf. Fig. 2(d), after which the intra-cavity field, reflecting the power coupled from the leading pulse edge, becomes high enough that a large number of free carriers is generated due to two-photon absorption. This changes the refractive index of the nanocavity and hence shifts the resonance towards shorter wavelength. The resonance shift as a function of time shown in Fig. 2(d) (black dashed curve) displays a maximum blue shift of $\sim 1 \mathrm{~nm}$ corresponding to the spectral separation between the transmission maximum and minimum of the Fano resonance (Fig. 1(c)). Therefore, the input pulse, which is centered at the peak of the resonance, encounters a large transmission suppression and the output pulse amplitude reaches its minimum. Because of this, a short pulse of $\sim 20 \mathrm{ps}$ (shown in Fig. 2(d) by blue solid line) is carved-out of the 93 ps input pulse. For comparison, we also simulated pulse carving using a structure based on Lorentzian resonance with the same Q-factor (red dashed line in Fig. 2(d)), however it displays only partial pulse carving under the same conditions. In contrast, the asymmetric Fano resonance shows complete pulse carving due to the steep slope between the transmission extrema. The spectrum of the pulse carved using the Fano resonance, obtained by taking the Fourier transform of the output temporal pulse, is depicted in Fig. 2(e), showing some spectral broadening.

Fig. 3 presents experimental and theoretical results for the output pulse at different power levels of the input pulse. In addition to the pulse shape, the input power level is important since it determines the magnitude of the resonance shift and hence the output pulse width and shape. The input (dashed line) is a 93 ps long Gaussian pulse and corresponding calculated output pulses (solid lines) are shown in Fig. 3(a) for power levels increasing from $-8 \mathrm{dBm}$ to $2 \mathrm{dBm}$. For clarity, the output power is normalized to unity at the peak. It is seen that at $0 \mathrm{dBm}$, a short pulse is generated with its center positioned at the leading edge of the input pulse. Fig. 3(b) shows the calculated output-toinput pulse peak power ratio for increasing average input power. This ratio decreases for increasing average input power because of the resonance shift induced at the leading edge of the input pulse, which results in suppressed transmission for a major part of the input pulse. Additionally, Fig. 3(b) shows the resonance shift for increasing average input power level. This is useful in order to calculate the required input power to shift from the transmission maximum of the Fano resonance to the minimum. Output pulses from the Fano carver measured using a sampling oscilloscope are shown in Fig. 3(c). The input pulse train, with 1 $\mathrm{GHz}$ repetition rate, is centered at the peak of the Fano resonance (Fig. 1(b)). The output pulse at an average input power of -14 $\mathrm{dBm}$ is shown in Fig. 3(c-i). This pulse has a width close to the 


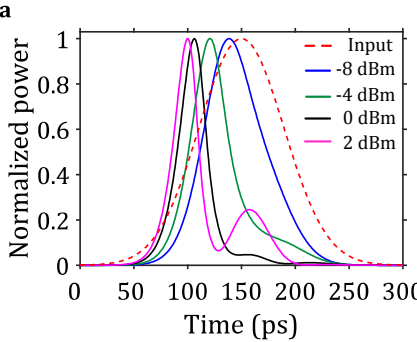

b
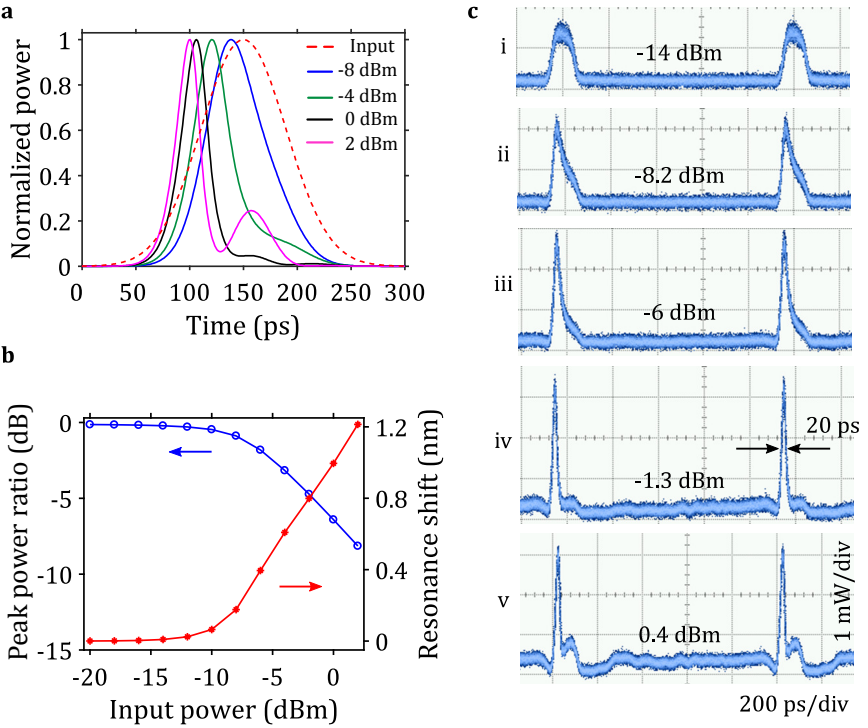

Fig. 3. Self-carving theory and experiment. (a) Calculated power vs time for a Gaussian input pulse and the carved output pulses for different average input power levels. (b) Calculated output-to-input pulse peak power ratio (blue) and resonance shift (red) for increasing input power. (c) Measured output pulses for average input power increasing from -14 $\mathrm{dBm}$ to $0.4 \mathrm{dBm}$ showing different stages of the self-carving process.

input pulse as the input power is low and cannot induce a shift of the resonance. For input power levels of $-8.2 \mathrm{dBm}$ and -6.0 $\mathrm{dBm}$, partial pulse carving is observed (ii and iii). In these cases, the resonance shift is not large enough to bring the minimum of the Fano resonance to the spectral position of the input signal. However, by gradually increasing the average input power of the pulse, it is possible to carve-out a pulse with width of $\sim 20$ ps as shown in Fig. 3(c-iv) for an input power of $-1.3 \mathrm{dBm}$. Furthermore, Fig. 3(a) predicts that for an input power of $2 \mathrm{dBm}$, a side peak appears. This is the result of a large resonance shift, where the minimum of the Fano resonance passes beyond the wavelength at which the input spectrum is centered and leading to increased transmission for latter portion of the input pulse. Fig. 3(c-v) shows the experimental observation of this side peak for input power of $0.4 \mathrm{dBm}$.

Further theoretical and experimental characteristics of selfcarved pulses are presented in Fig. 4. Sampling oscilloscope traces of 500 ps input pulses (top) and self-carved pulses (bottom) both at $400 \mathrm{MHz}$ repetition rate are shown in Fig. 4(a). When the input power is around $0 \mathrm{dBm}$, a resonance shift to the minimum of the Fano resonance is possible and hence a short pulse of $\sim 30$ ps is carved-out of the input pulse. The inset shows a zoomed-in trace of the output pulse. Measured optical spectra of the input and output pulses are presented in Fig. 4(b). The full width at half maximum (FWHM) of the pulses is $\sim 0.028$ $\mathrm{nm}$, corresponding to a bandwidth of $\sim 3.48 \mathrm{GHz}$ at the central wavelength of $1553.86 \mathrm{~nm}$. Note that the measurements are not limited by the resolution of the optical spectrum analyzer (Ando AQ6317B) which is $0.01 \mathrm{~nm}$. The time-bandwidth product (TBP) of the carved pulse is $3.48 \mathrm{GHz} \times 30 \mathrm{ps}=0.104$. In comparison, a Gaussian chirp-free pulse has a FWHM TBP of $\sim 0.44$ [19]. To validate the small TBP of the carved pulse, we performed theoretical investigations using CMT. Fig. 4(c) shows a 500 ps
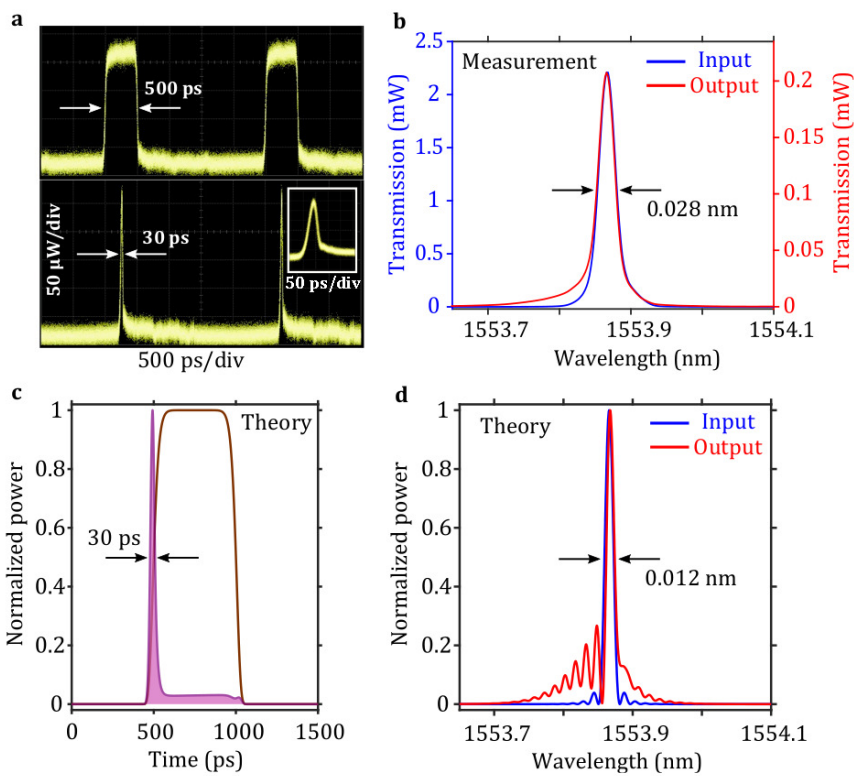

Fig. 4. Temporal and spectral characterization of self-carving for 500 ps input pulses. (a) Sampling oscilloscope trace of a 500 ps pulse (top) and carved output (bottom) having a width of $\sim 30 \mathrm{ps}$ for $0 \mathrm{dBm}$ input power. The inset shows a zoomin view of the output pulse. (b) Measured optical spectra for input and carved pulses shown in (a). (c) Calculated temporal profile of the input and carved pulse. (d) Simulated optical spectra of the pulses in (c).

long super Gaussian flattop input pulse of order 6 and the corresponding self-carved temporal pulse. A super Gaussian pulse is chosen for better agreement with the experiment. The temporal profile of the self-carved pulse shows generation of a short pulse located at the edge of the input pulse. It can also be observed that the carved pulse has a pulse tail which is not completely suppressed. The power spectrum of these pulses is presented in Fig. 4(d). Asymmetric spectral broadening is observed for the self-carved pulse similar to the measured spectrum shown in Fig. 4(b). This is because of the mechanism of pulse carving which suppresses the trailing edges of the temporal pulse with less influence on the leading edge. Moreover, the theoretical FWHM for both the input and self-carved output is found to be $\sim 0.012 \mathrm{~nm}$ and the corresponding TBPs are $\sim 0.836$ and $\sim 0.046$ for the input and output pulses, respectively. The small TBP of the output pulse is enabled by the presence of a significant temporal pulse tail which keeps the FWHM spectral bandwidth to remain close to that of input pulse. Further optimization of the slope and transmission contrast of the Fano resonance should be done for complete suppression of pulse tails in which case considerable spectral broadening is expected for the carved pulse.

One possible application of such self-carving is for reducing the time slot or duty cycle of return-to-zero on-off keying (RZOOK) modulated signals. Fig. 5(a) shows the eye diagram of a $2 \mathrm{Gbit} / \mathrm{s}$ input signal. It is modulated by a pseudo-random bit sequence of length of $2^{15}-1$. It shows that a single channel occupies a time slot of $\sim 150 \mathrm{ps}$ and has a pulse width around 100 ps. With the implementation of the Fano resonance based self-carver, it is possible to reduce the time slot to $\sim 60 \mathrm{ps}$ and the width to $\sim 30$ ps (Fig. 5(b)). Bit-error-ratio (BER) performance measurements for the reference input and carved output signals 


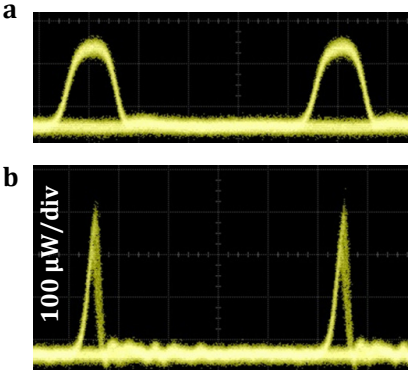

$100 \mathrm{ps} / \mathrm{div}$

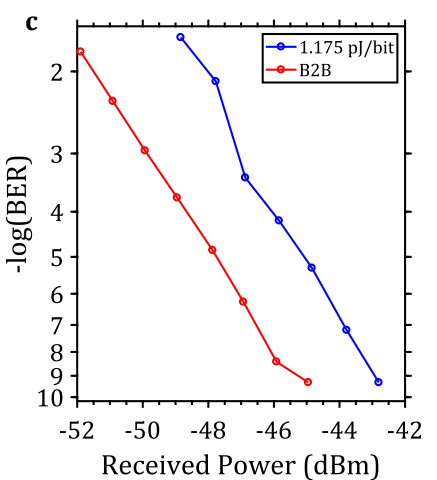

Fig. 5. Low duty cycle RZ signal generation. (a) Eye diagram of 2 Gbit/s RZ-OOK modulated input signal with pulse width of $\sim 100$ ps and duty cycle of $\sim 20 \%$. (b) Eye diagram of carved output RZ signal with pulse width of $\sim 30$ ps and duty cycle of $\sim 6 \%$. (c) BER performance measurements for the input signal and self-carved low duty cycle RZ signal.

as function of received power are shown in Fig. 5(c). With an energy consumption down to $1.175 \mathrm{pJ} / \mathrm{bit}$, the carved signal clearly shows error-free performance with a power penalty of $\sim 2 \mathrm{~dB}$ at the BER of $10^{-9}$ compared to a 2 Gbit/s input signal measured back-to-back (B2B). The power penalty reflects the additional power required to compensate for the peak power reduction of the carved pulse (cf. Fig. 3(b)). These experiments demonstrate that the simple scheme of our Fano structure can be implemented in optical time domain multiplexing systems for achieving high speed on-chip data transmission.
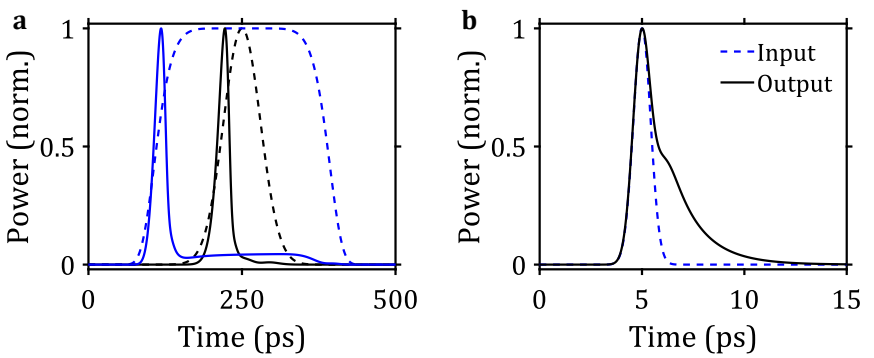

Fig. 6. Limit of pulse carving. (a) Pulse carving for 50 ps (dashed black) and 118 ps (dashed blue) input pulses to $\sim 20.8$ ps pulses (solid lines). (b) Device output (solid black) for 1 ps input pulse (dashed blue) with spectral width of $(\sim 3.5 \mathrm{~nm})$ at $1550 \mathrm{~nm}$.

Fig. 6(a) shows that for input pulses with similar rise time ( $\sim 35$ ps) but different widths, the output carved pulses have very similar widths of $\sim 20.8 \mathrm{ps}$. This is because once carrier saturation is achieved in the nanocavity, the resonance will stay at the blue shifted spectral location for the duration of the input pulse, which enables the Fano resonance to carve away the remainder part of the pulse regardless of its width. On the other hand, for input pulses having spectral width larger than the spectral separation of the extrema of the Fano resonance $(\sim 1 \mathrm{~nm})$, pulse carving is not effective, as depicted in Fig. 6(b) illustrating the presence of a significant pulse tail corresponding to photons decaying from the cavity hence broadening the output. Therefore, for a Fano resonance with $1 \mathrm{~nm}$ separation between the resonance extrema, the shortest input pulse that can be carved is $\sim 5$ ps.

In conclusion, we proposed and demonstrated a compact and simple device that carves a short pulse out of a long input pulse, without requiring any external modulation. The device relies on the presence of a sharp asymmetric Fano resonance, realized in an InP PhC membrane structure, in combination with a nanocavity-enhanced nonlinear optical effect that shifts the Fano resonance to modulate the input pulse itself. Currently, these self-carving experiments are limited to pulse repetition rates less than $5 \mathrm{GHz}$. This limitation is mainly due to the requirement that a complete recovery of the resonance shift should occur before the next pulse arrives at the nanocavity. For our $\mathrm{InP}$ based PhC nanocavities, this recovery time scale is mainly governed by diffusion of charge carriers and carrier recombination rate [16]. Therefore, to increase the speed, one needs to find ways to increase the rate at which carriers recombine or leave the nanocavity region. We believe that with implementation of carrier sweep out mechanisms such as by applying a reverse bias voltage across the nanocavity region, higher repetition rates would be possible by shifting from a diffusion-limited regime to a carrier drift limited regime.

\section{FUNDING}

Financial support from Villum Fonden via the NAnophotonics for TErabit Communications (NATEC) center of excellence under grant number 8692 is gratefully acknowledged.

\section{REFERENCES}

1. A. E. Miroshnichenko, S. Flach, and Y. S. Kivshar, Rev. Mod. Phys. 82, 2257 (2010).

2. S. Fan, Appl. Phys. Lett. 80, 908 (2002).

3. M. F. Limonov, M. V. Rybin, A. N. Poddubny, and Y. S. Kivshar, Nature Photonics 11, 543 (2017).

4. Y. Yu, H. Hu, L. K. Oxenløwe, K. Yvind, and J. Mork, Opt. Lett. 40, 2357 (2015).

5. Y. Yu, W. Xue, E. Semenova, K. Yvind, and J. Mork, Nat. Photon. 11, 81 (2017).

6. Y. Yu, Y. Chen, H. Hu, W. Xue, K. Yvind, and J. Mork, Laser \& Photon. Rev. 9, 241 (2015).

7. B. Luk'yanchuk, N. I. Zheludev, S. A. Maier, N. J. Halas, P. Nordlander, H. Giessen, and C. T. Chong, Nat. Mater. 9, 707 (2010).

8. D. A. Miller, Proceedings of the IEEE 97, 1166 (2009).

9. D. Breuer and K. Petermann, IEEE Photon. Technol. Lett. 9, 398 (1997).

10. X. Zhao, C. Lou, H. Zhou, D. Lu, and L. Huo, Opt. Express 18, 23657 (2010).

11. Z. Zhang and M. Qiu, Optics Express 12, 3988 (2004).

12. M. Heuck, P. T. Kristensen, Y. Elesin, and J. Mørk, Opt. lett. 38, 2466 (2013).

13. D. A. Bekele, Y. Yu, H. Hu, Y. Ding, A. Sakanas, L. Ottaviano, E. Semenova, L. K. Oxenløwe, K. Yvind, and J. Mork, Proc. SPIE 10345, 1034501 (2017).

14. C. Husko, A. De Rossi, S. Combrié, Q. V. Tran, F. Raineri, and C. W. Wong, Applied Physics Letters 94, 021111 (2009).

15. K. Nozaki, T. Tanabe, A. Shinya, S. Matsuo, T. Sato, H. Taniyama, and M. Notomi, Nat. Photon. 4, 477 (2010).

16. Y. Yu, E. Palushani, M. Heuck, N. Kuznetsova, P. T. Kristensen, S. Ek, D. Vukovic, C. Peucheret, L. K. Oxenløwe, S. Combrié, A. de Rossi, K. Yvind, and J. Mørk, Opt. Express 21, 31047 (2013).

17. W. Suh, Z. Wang, and S. Fan, IEEE J. Quant. Electron. 40, 1511 (2004).

18. P. T. Kristensen, J. R. de Lasson, M. Heuck, N. Gregersen, and J. Mørk, J. Light. Technol. 35, 4247 (2017).

19. A. Weiner, Ultrafast optics (John Wiley \& Sons, 2011). 


\section{FULL REFERENCES}

1. A. E. Miroshnichenko, S. Flach, and Y. S. Kivshar, "Fano resonances in nanoscale structures," Rev. Mod. Phys. 82, 2257 (2010).

2. S. Fan, "Sharp asymmetric line shapes in side-coupled waveguidecavity systems," Appl. Phys. Lett. 80, 908-910 (2002).

3. M. F. Limonov, M. V. Rybin, A. N. Poddubny, and Y. S. Kivshar, "Fano resonances in photonics," Nature Photonics 11, 543-554 (2017).

4. Y. Yu, H. Hu, L. K. Oxenløwe, K. Yvind, and J. Mork, "Ultrafast alloptical modulation using a photonic-crystal Fano structure with broken symmetry," Opt. Lett. 40, 2357-2360 (2015).

5. Y. Yu, W. Xue, E. Semenova, K. Yvind, and J. Mork, "Demonstration of a self-pulsing photonic crystal Fano laser," Nat. Photon. 11, 81-84 (2017).

6. Y. Yu, Y. Chen, H. Hu, W. Xue, K. Yvind, and J. Mork, "Nonreciprocal transmission in a nonlinear photonic-crystal Fano structure with broken symmetry," Laser \& Photon. Rev. 9, 241-247 (2015).

7. B. Luk'yanchuk, N. I. Zheludev, S. A. Maier, N. J. Halas, P. Nordlander, H. Giessen, and C. T. Chong, "The Fano resonance in plasmonic nanostructures and metamaterials," Nat. Mater. 9, 707-715 (2010).

8. D. A. Miller, "Device requirements for optical interconnects to silicon chips," Proceedings of the IEEE 97, 1166-1185 (2009).

9. D. Breuer and K. Petermann, "Comparison of NRZ- and RZ-modulation format for 40-Gb/s TDM standard-fiber systems," IEEE Photon. Technol. Lett. 9, 398-400 (1997).

10. X. Zhao, C. Lou, H. Zhou, D. Lu, and L. Huo, "Optical regenerative $\mathrm{NRZ}$ to $\mathrm{RZ}$ format conversion based on cascaded lithium niobate modulators," Opt. Express 18, 23657-23663 (2010).

11. Z. Zhang and M. Qiu, "Small-volume waveguide-section high q microcavities in 2d photonic crystal slabs," Optics Express 12, 3988-3995 (2004).

12. M. Heuck, P. T. Kristensen, Y. Elesin, and J. Mørk, "Improved switching using Fano resonances in photonic crystal structures," Opt. lett. 38, 2466-2468 (2013).

13. D. A. Bekele, Y. Yu, H. Hu, Y. Ding, A. Sakanas, L. Ottaviano, E. Semenova, L. K. Oxenløwe, K. Yvind, and J. Mork, "Photonic crystal Fano resonances for realizing optical switches, lasers, and non-reciprocal elements," Proc. SPIE 10345, 1034501-1034507 (2017).

14. C. Husko, A. De Rossi, S. Combrié, Q. V. Tran, F. Raineri, and C. W. Wong, "Ultrafast all-optical modulation in gaas photonic crystal cavities," Applied Physics Letters 94, 021111 (2009).

15. K. Nozaki, T. Tanabe, A. Shinya, S. Matsuo, T. Sato, H. Taniyama, and M. Notomi, "Sub-femtojoule all-optical switching using a photoniccrystal nanocavity," Nat. Photon. 4, 477-483 (2010).

16. Y. Yu, E. Palushani, M. Heuck, N. Kuznetsova, P. T. Kristensen, S. Ek, D. Vukovic, C. Peucheret, L. K. Oxenløwe, S. Combrié, A. de Rossi, K. Yvind, and J. Mørk, "Switching characteristics of an InP photonic crystal nanocavity: Experiment and theory," Opt. Express 21, 3104731061 (2013).

17. W. Suh, Z. Wang, and S. Fan, "Temporal coupled-mode theory and the presence of non-orthogonal modes in lossless multimode cavities," IEEE J. Quant. Electron. 40, 1511-1518 (2004).

18. P. T. Kristensen, J. R. de Lasson, M. Heuck, N. Gregersen, and J. Mørk, "On the theory of coupled modes in optical cavity-waveguide structures," J. Light. Technol. 35, 4247-4259 (2017).

19. A. Weiner, Ultrafast optics (John Wiley \& Sons, 2011). 